\title{
Unitarity of superstring field theory
}

\section{Ashoke Sen}

Harish-Chandra Research Institute, Chhatnag Road, Jhusi, Allahabad 211019, India

Homi Bhabha National Institute,

Training School Complex, Anushakti Nagar, Mumbai 400085, India

E-mail: sen@mri.ernet.in

ABstract: We complete the proof of unitarity of (compactified) heterotic and type II string field theories by showing that in the cut diagrams only physical states appear in the sum over intermediate states. This analysis takes into account the effect of mass and wave-function renormalization, and the possibility that the true vacuum may be related to the perturbative vacuum by small shifts in the string fields.

KEYwords: String Field Theory, Superstrings and Heterotic Strings

ARXIV EPRINT: 1607.08244 


\section{Contents}

1 Introduction 1

2 The propagator $\quad 2$

$\begin{array}{llr}3 & \text { Unitarity } & 8\end{array}$

4 Discussions $\quad 12$

\section{Introduction}

In a previous paper [1] we derived the Cutkosky rules for superstring field theory under the assumption that the action for string field theory is real. This assumption was proved later [2]. Cutkosky rules derived in [1] establish that the T-matrix - related to the Smatrix via the relation $S=1-i T$ - satisfies the relation $i\left(T-T^{\dagger}\right)=T^{\dagger}|n\rangle\langle n| T$, where the sum over $|n\rangle$ runs over all states in the Siegel gauge. This would establish unitarity of the theory if all states in the Siegel gauge were physical states, However since string field theory is a gauge theory, Cutkosky rules do not automatically prove unitarity. A cut propagator representing $|n\rangle\langle n|$, besides propagating physical on-shell intermediate states, also has unphysical and pure gauge states. Therefore in order to prove unitarity we need to prove that the sum over intermediate states in a cut diagram receives contribution from only the physical on-shell states, and the contribution from all other states cancel. This is what we shall show in this paper.

Earlier attempts $[3,4]$ to prove unitarity of superstring theory in the covariant formulation relied on proving equivalence to light-cone string field theory $[5,6]$. However since light-cone superstring field theory encounters contact term divergences [7-10], it is not clear if this can be lifted to a valid proof after taking into account the various subtleties of the covariant formulation described in [11]. Some recent attempts to circumvent this difficulty can be found in [12]. Another systematic procedure for computing the imaginary part of the string theory amplitude is the $i \epsilon$ prescription of $[13,14]$, but it is not clear at this stage how this can be used to prove unitarity of the amplitude.

The rest of the paper is organized as follows. In section 2 we derive some useful properties of the quantum corrected propagator of string field theory and residues at its poles. In section 3 we use the Ward identities of string field theory to show that only physical states contribute in the sum over intermediate states in a cut diagram. In section 4 we discuss some open problems. 


\section{The propagator}

The main tool in our analysis will be the full propagator computed from the one particle irreducible (1PI) effective action of superstring field theory. We shall follow the conventions of [15], and begin by collecting some basic results in 1PI effective string field theory as reviewed in [15]. This will be followed by a review of some basic results in Cutkosky rules derived in [1]. Finally we shall combine these results to derive the general form of the contribution from a cut propagator as given in (2.30).

We shall work with the heterotic string theory for simplicity and will describe the generalization to type II string theories at the end of the section. We define $\mathcal{H}_{T}$ to be the subspace of GSO even states in the matter ghost conformal field theory satisfying

$$
b_{0}^{-}|s\rangle=0, \quad L_{0}^{-}|s\rangle=0, \quad \text { for }|s\rangle \in \mathcal{H}_{T} .
$$

Here $b_{n}, \bar{b}_{n}, c_{n}, \bar{c}_{n}$ are the modes of the usual $b, \bar{b}, c, \bar{c}$ ghost fields, $L_{n}, \bar{L}_{n}$ are the total Virasoro generators, and

$$
b_{0}^{ \pm}=b_{0} \pm \bar{b}_{0}, \quad c_{0}^{ \pm}=\frac{1}{2}\left(c_{0} \pm \bar{c}_{0}\right), \quad L_{0}^{ \pm}=L_{0} \pm \bar{L}_{0} .
$$

$\mathcal{H}_{n}$ will denote the subspace of states in $\mathcal{H}_{T}$ carrying picture number $n$.

Even though we follow closely the formalism described in [15], there are two ways in which the action that we consider differs from the one analyzed in [15]:

1. We shall implicitly assume that the 1PI effective action we use comes from the sum of 1PI diagrams of the action described in [16] so that the Cutkosky rules hold [1]. This means that the class of actions we shall consider will be more restrictive than the ones used in [15]. But this does not prevent us from using the results derived in [15] since the latter describes a more general class of theories.

2. In the analysis of [15] we had restricted the interacting string field to carry ghost number 2 and picture numbers -1 or $-1 / 2 .{ }^{1}$ Here we shall keep the form of the action unchanged but allow the string field to carry all possible ghost numbers since we want to include in our analysis not only the matter fields but also the ghost fields as external states. ${ }^{2}$ It is easy to verify that the results of [15] that we shall be using, namely eq. (2.8) below for the propagator and eq. (3.1) below for the truncated Green's function, are valid for these more general string fields.

The kinetic operator $\widehat{Q}_{B}$ of $1 \mathrm{PI}$ effective string field theory around the quantum corrected vacuum, and a related operator $\widetilde{Q}_{B}$ introduced in [15] will play special roles in our analysis. $\widehat{Q}_{B}$ and $\widetilde{Q}_{B}$ are operators of ghost number 1 , acting respectively on the states in

$$
\widehat{\mathcal{H}}_{T} \equiv \mathcal{H}_{-1} \oplus \mathcal{H}_{-1 / 2} \quad \text { and } \quad \widetilde{\mathcal{H}}_{T} \equiv \mathcal{H}_{-1} \oplus \mathcal{H}_{-3 / 2},
$$

\footnotetext{
${ }^{1}$ The formulation of the theory given in [16] also requires us to introduce a free string field taking value in $\mathcal{H}_{-1} \oplus \mathcal{H}_{-3 / 2}$, but this will not play any role in our analysis.

${ }^{2}$ The corresponding action will describe the '1PI master action' and will satisfy the classical master equation like the classical master action $[16,17]$. As in $[16,17]$, the master action is obtained from the original action by relaxing the constraint on ghost number of the string field, but keeping the form of the action the same.
} 
producing states in $\widehat{\mathcal{H}}_{T}$ and $\widetilde{\mathcal{H}}_{T}$ respectively. $\widehat{Q}_{B}$ and $\widetilde{Q}_{B}$ have the form

$$
\widehat{Q}_{B}=Q_{B}+\mathcal{G} K, \quad \widetilde{Q}_{B}=Q_{B}+K \mathcal{G} .
$$

Here $Q_{B}$ is the nilpotent BRST operator. $K$ is some operator that acts on states in $\widehat{\mathcal{H}}_{T}$ and produces states in $\widetilde{\mathcal{H}}_{T}$. It is related to the $1 \mathrm{PI}$ two point function and can be computed using perturbation theory. $\mathcal{G}$ is the identity operator in the Neveu-Schwarz (NS) sector and the zero mode of the picture changing operator $(\mathrm{PCO})$ in the Ramond (R) sector, and satisfies

$$
\left[\mathcal{G}, b_{0}^{ \pm}\right]=0, \quad\left[\mathcal{G}, L_{0}^{ \pm}\right]=0, \quad\left[\mathcal{G}, Q_{B}\right]=0 .
$$

$\widehat{Q}_{B}$ and $\widetilde{Q}_{B}$ satisfy

$$
\widehat{Q}_{B}^{2}=0, \quad \widetilde{Q}_{B}^{2}=0,
$$

and

$$
\begin{aligned}
& \langle A| c_{0}^{-} \widehat{Q}_{B}=(-1)^{\gamma_{A}}\left\langle\widetilde{Q}_{B} A\right| c_{0}^{-} \quad \text { for }|A\rangle \in \widetilde{\mathcal{H}}_{T}, \\
& \langle B| c_{0}^{-} \widetilde{Q}_{B}=(-1)^{\gamma_{B}}\left\langle\widehat{Q}_{B} B\right| c_{0}^{-} \quad \text { for }|B\rangle \in \widehat{\mathcal{H}}_{T},
\end{aligned}
$$

where $\gamma_{A}$ and $\gamma_{B}$ are grassmannalities of $A$ and $B$, and $\left\langle\widetilde{Q}_{B} A\right|$ and $\left\langle\widehat{Q}_{B} B\right|$ are respectively the BPZ conjugates of $\widetilde{Q}_{B}|A\rangle$ and $\widehat{Q}_{B}|B\rangle$. It follows from (2.4) that $\widehat{Q}_{B} \mathcal{G}=\mathcal{G} \widetilde{Q}_{B}$.

While the interacting string field takes value in $\widehat{\mathcal{H}}_{T}$, Siegel gauge condition further restricts the string field to be annihilated by $b_{0}^{+}$. The full Siegel gauge propagator $\Delta$ for the interacting string field of the 1PI effective action was constructed in [15]. It acts on states in $c_{0}^{-} \widetilde{\mathcal{H}}_{T}$ and produces states in $\widehat{\mathcal{H}}_{T}$, and has the form

$$
\Delta=\beta \mathcal{G}\left(L_{0}^{+}+b_{0}^{+} K \mathcal{G}\right)^{-1} b_{0}^{+} b_{0}^{-}=\beta \mathcal{G} b_{0}^{+}\left(L_{0}^{+}+K \mathcal{G} b_{0}^{+}\right)^{-1} b_{0}^{-} \quad \text { acting on states in } c_{0}^{-} \widetilde{\mathcal{H}}_{T} .
$$

Here $\beta$ is a constant that depends on the normalization of the action and includes a factor of $i$ for Lorentzian signature space-time background. $\Delta$ satisfies

$$
\begin{aligned}
b_{0}^{+} \Delta & =0, & & \Delta b_{0}^{+}=0, \\
\widehat{Q}_{B} \Delta c_{0}^{-}+\Delta c_{0}^{-} \widetilde{Q}_{B} & =\beta \mathcal{G} & & \text { acting on states in } \widetilde{\mathcal{H}}_{T} .
\end{aligned}
$$

Construction of $\Delta$ requires inverting the operator $L_{0}^{+}+b_{0}^{+} K \mathcal{G}$ which is an infinite dimensional matrix. At generic momentum we can evaluate $\Delta$ using perturbation theory in $K$ leading to a sum over Feynman diagrams contributing to the off-shell two point function. However when the momentum is close to a value where $L_{0}^{+}$vanishes for some states, perturbation theory breaks down since diagrams of higher order will have poles of higher order. In this case a systematic procedure for computing $\Delta$ was described in [18]. The strategy is to work at some fixed mass ${ }^{2}$ level $L$ (defined by the momentum independent contribution to $L_{0}^{+}$) and 'integrate out' the contribution to $\Delta$ from fields in the other mass levels. The latter operation can be carried out perturbatively. This generates a finite dimensional matrix in the space of states at the mass ${ }^{2}$ level $L$ which can then be inverted explicitly. More explicitly, this corresponds to using the identity

$$
\left(\begin{array}{ll}
A & B \\
C & D
\end{array}\right)^{-1}=\left(\begin{array}{cc}
\left(A-B D^{-1} C\right)^{-1} & -\left(A-B D^{-1} C\right)^{-1} B D^{-1} \\
-D^{-1} C\left(A-B D^{-1} C\right)^{-1} & D^{-1}+D^{-1} C\left(A-B D^{-1} C\right)^{-1} B D^{-1}
\end{array}\right)
$$




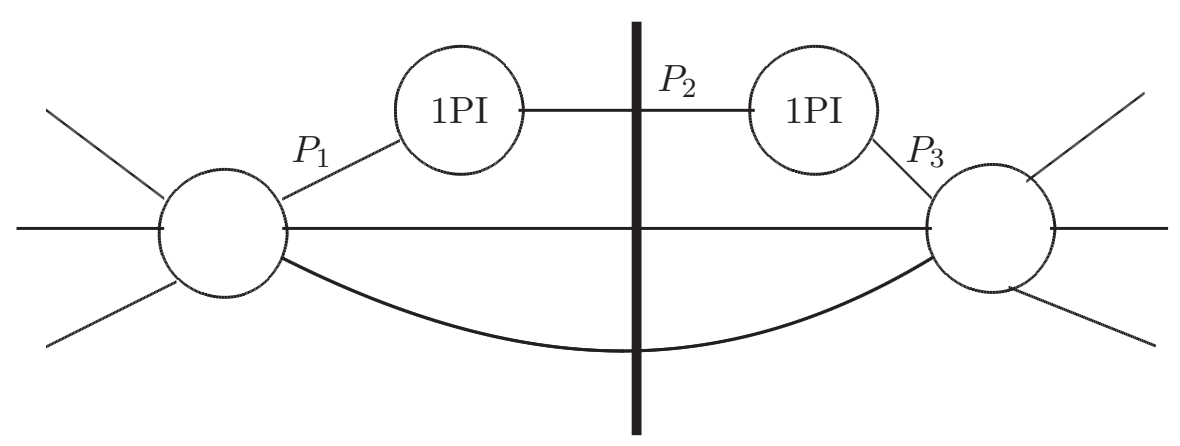

Figure 1. A problematic cut diagram.

where $A$ and $D$ are square matrices and $B$ and $C$ are rectangular matrices. In our case $A$ denotes the kinetic operator at mass ${ }^{2}$ level $L$ and is a finite dimensional matrix, $D$ is the kinetic operator for all mass ${ }^{2}$ levels other than $L$ and $B$ and $C$ are the mixing matrices between mass ${ }^{2}$ level $L$ and mass ${ }^{2}$ level other than $L . D^{-1}$ can be computed perturbatively to any given order, and we compute $\left(A-B D^{-1} C\right)^{-1}$ by exact matrix inversion. This matrix then can be used e.g. to find the poles of $\Delta$ in the $k^{2}$ plane for $-k^{2}$ near mass ${ }^{2}$ level $L$.

We now briefly review the results of [1]. The analysis of [1] tells us that the string field theory amplitudes obey the Cutkosky cutting rules. These rules may be summarized as follows. ${ }^{3}$ If we draw a Feynman diagram with the incoming states on the left and the outgoing states on the right, then the contribution to $i\left(T-T^{\dagger}\right)$ is given by the sum over all cuts of the diagram where a cut is a line through the diagram separating the incoming states from the outgoing states. The rule for computing a cut diagram is to replace a cut internal propagator $-i\left(k^{2}+m_{0}^{2}\right)^{-1}$ by $2 \pi \delta\left(k^{2}+m_{0}^{2}\right) \theta\left(k^{0}\right)$. Here $m_{0}$ is the tree level mass, $k$ is the momentum flowing through the cut propagator from the left to the right and $\theta$ denotes step function. Furthermore, the amplitude to the right of the cut is hermitian conjugated. However, naive application of this result will give divergent result from cut diagrams of the form shown in figure 1. The cut passing through the propagator $P_{2}$ forces the momentum passing through this to be on-shell, but this also forces the momentum passing through the uncut propagators $P_{1}$ and $P_{3}$ to be on-shell, making them diverge. The remedy suggested in $[19,20]$ is to sum over all cuts of a propagator to express the result as the hermitian part of the full propagator. It is simplest to illustrate this through a scalar field propagator. Let us suppose that the full quantum corrected propagator has the form

$$
\Delta=-i\left(k^{2}+m_{0}^{2}+\Gamma(k)-i \epsilon\right)^{-1},
$$

where $m_{0}$ is the tree level mass, $k$ is the momentum flowing from the left to the right, and $-i \Gamma(k)$ represents the contribution from the 1PI two point function. Then the sum over

\footnotetext{
${ }^{3}$ Here we are considering the Feynman diagrams of superstring field theory expanded around the shifted background, and not that of 1PI effective string field theory. For this reason the vertices are hermitian, we need to include loop diagrams, and Cutkosky rules hold. If we use the 1PI effective action to compute the amplitude, then there are no loop diagrams, but the verices will be complex and part of the contribution to the ant-hermitian part of an amplitude will arise from the anti-hermitian part of the vertices.
} 


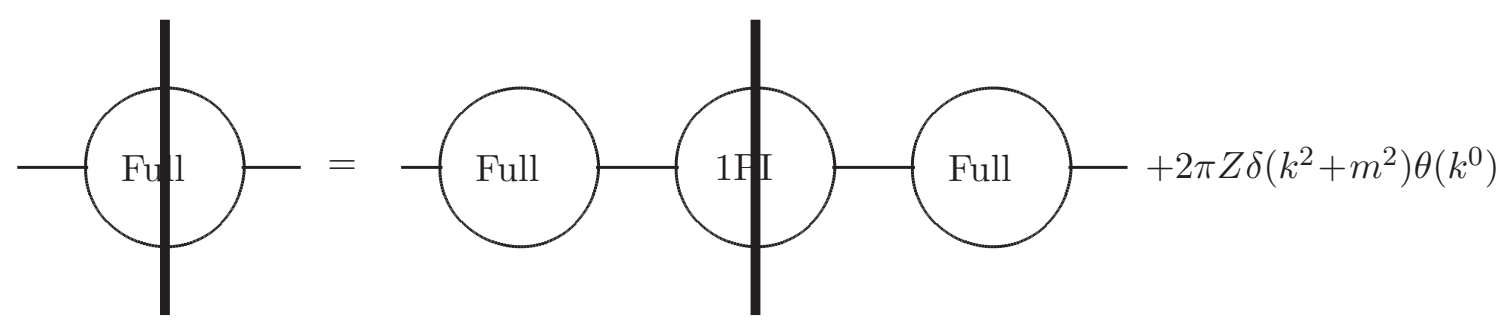

Figure 2. Diagrammatic representation of (2.13)-(2.15). The left hand side represents (2.13), the first term on the right hand side represents (2.14) and the second term on the right hand side represents (2.15).

all cuts of the propagator can be expressed as

$$
-i\left[\left(k^{2}+m_{0}^{2}+\Gamma(k)-i \epsilon\right)^{-1}-\left(k^{2}+m_{0}^{2}+\Gamma(k)^{*}+i \epsilon\right)^{-1}\right] \theta\left(k^{0}\right) .
$$

There are three cases to be considered. If for the value of $k$ of interest $\Gamma(k)$ has an imaginary part, then we can ignore the $i \epsilon$ term and express (2.13) as

$$
-i\left(k^{2}+m_{0}^{2}+\Gamma(k)\right)^{-1} i\left(\Gamma(k)-\Gamma(k)^{*}\right) \theta\left(k^{0}\right) i\left(k^{2}+m_{0}^{2}+\Gamma(k)^{*}\right)^{-1} .
$$

If $\Gamma(k)$ is real and $k^{2}+m_{0}^{2}+\Gamma(k)$ is away from 0 , then $(2.13)$ vanishes. Finally if $\Gamma(k)$ is real and the full propagator has a pole on the real $k^{2}$ axis at $k^{2}+m^{2}=0$ with residue $-i Z$, then (2.13) behaves as

$$
2 \pi Z \delta\left(k^{2}+m^{2}\right) \theta\left(k^{0}\right),
$$

near $k^{2}+m^{2}=0$. Therefore (2.13) may be expressed as the sum of (2.14) and (2.15). This can be represented diagrammatically as in figure 2 .

For the full superstring field theory this has the following consequence. Let us suppose that the full propagator $\Delta$ has a pole at $k^{2}+m^{2}=0$. Then near $k^{2}=-m^{2}$ we have

$$
\Delta=-i\left(k^{2}+m^{2}-i \epsilon\right)^{-1} \Delta_{0}+\text { non-singular } .
$$

The $i \epsilon$ determines the side of the integration contour on which the pole lies [1]. Even though $\Delta$ is an infinite dimensional matrix, $\Delta_{0}$ is a matrix of finite rank since for given momentum we expect only a finite number of states for which the propagator develops a pole at $k^{2}=-m^{2}$. The rules for computing the contribution from a cut propagator can be summarized as follows:

1. If $m^{2}$ is real then the corresponding cut propagator should be replaced by

$$
2 \pi \delta\left(k^{2}+m^{2}\right) \theta\left(k^{0}\right) \Delta_{0},
$$

where $k$ is the momentum carried by the cut propagator from the left side of the cut to the right side. Furthermore we do not include any virtual self-energy corrections on either side of a cut propagator, e.g. the diagrams of the type shown in figure 3(a) are not allowed since their contribution has already been included in the $\Delta_{0}$ factor 


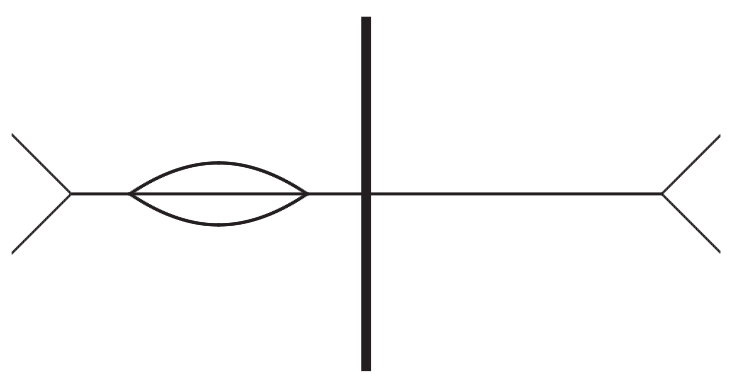

(a)

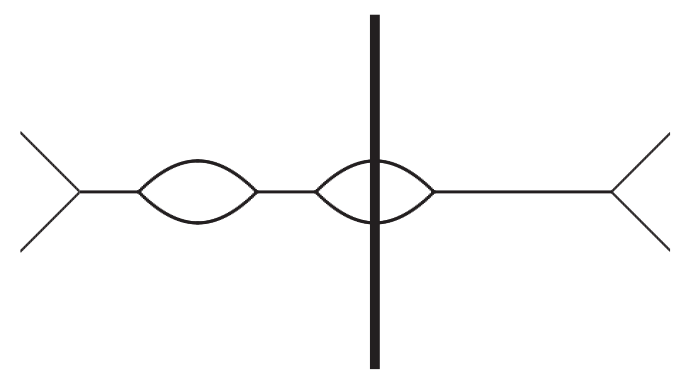

(b)

Figure 3. Figure (a) shows the example of a disallowed cut diagram and figure (b) shows the example of an allowed cut diagram. In both examples the thick vertical line denotes the cut.

in (2.17) and in the use of the renormalized mass $m$. However a cut can pass through a self energy diagram, e.g. a diagram of the form shown in figure 3(b) is allowed. These diagrams capture the imaginary part of the propagator other than the pole contribution (2.17), as represented by (2.14).

2. If $m^{2}$ has an imaginary part, i.e. if the pole corresponds to an unstable particle, then we do not have the contribution (2.17). This is consistent with the fact that unstable particles are not genuine asymptotic states and should not appear in the sum over intermediate states in a unitarity relation. However cuts passing through the self energy diagrams, like the ones shown in figure 3(b) are still allowed.

3. If the momentum $k$ carried by a virtual uncut propagator is near a pole of the propagator, we must use the resummed propagator that includes repeated insertion of one particle irreducible (1PI) self-energy diagrams on the propagator. For our analysis this means that near $k^{2}+m^{2}=0$ we should use the propagator $\Delta$ constructed using (2.8), (2.11) which already has resummation built into it. ${ }^{4}$

Therefore for analyzing cut diagrams we need to focus on the properties of $\Delta_{0}$ associated with the poles that occur at real momenta. Multiplying both sides of (2.9), (2.10) by $k^{2}+m^{2}$ and taking the limit $k^{2} \rightarrow-m^{2}$, we get

$$
\begin{gathered}
b_{0}^{+} \Delta_{0}=0, \quad \Delta_{0} b_{0}^{+}=0, \\
\widehat{Q}_{B} \Delta_{0} c_{0}^{-}+\Delta_{0} c_{0}^{-} \widetilde{Q}_{B}=0 .
\end{gathered}
$$

Multiplying (2.19) by $b_{0}^{+}$from left/right and using (2.18) we get

$$
b_{0}^{+} \widehat{Q}_{B} \Delta_{0} c_{0}^{-}=0, \quad \Delta_{0} c_{0}^{-} \widetilde{Q}_{B} b_{0}^{+}=0 .
$$

\footnotetext{
${ }^{4}$ This rule is particularly important for unstable particles as can be illustrated using the example of figure 3(b). If the intermediate single particle state represented by the horizontal line corresponds to an unstable particle, it is kinematically possible for momentum flowing through the horizontal line to be near its classical on-shell value, and repeated insertion of self-energy diagrams on this will generate divergences of arbitrarily high order. Therefore we must use the resummed propagator for which we only have first order pole and the pole is shifted away from the real axis. In the limit of zero string coupling, the combined contribution from the resummed propagators on two sides of the cut and the contribution from the cut 1PI two point function approaches the delta function contribution given in (2.17).
} 
Let us now use a general ansatz

$$
\Delta_{0}=\sum_{m=1}^{R}\left|\Phi_{m}\right\rangle\left\langle\Psi_{m}\right|
$$

where $R$ is the rank of $\Delta_{0}$ and $\left\{\left|\Phi_{m}\right\rangle\right\}$ and $\left\{\left\langle\Psi_{m}\right|\right\}$ are a set of linearly independent states. Since $\Delta_{0}$ acts on states in $c_{0}^{-} \widetilde{\mathcal{H}}_{T}$ to produce states in $\widehat{\mathcal{H}}_{T}$, and BPZ inner product pairs states in $\widehat{\mathcal{H}}_{T}$ with states in $c_{0}^{-} \widetilde{\mathcal{H}}_{T}$, we have

$$
\left|\Phi_{m}\right\rangle \in \widehat{\mathcal{H}}_{T}, \quad\left|\Psi_{m}\right\rangle \in \widehat{\mathcal{H}}_{T} .
$$

Eqs. (2.18), (2.20) now give

$$
b_{0}^{+}\left|\Phi_{m}\right\rangle=0, \quad\left\langle\Psi_{m}\left|b_{0}^{+}=0, \quad b_{0}^{+} \widehat{Q}_{B}\right| \Phi_{m}\right\rangle=0, \quad\left\langle\Psi_{m}\right| c_{0}^{-} \widetilde{Q}_{B} b_{0}^{+}=0 \Rightarrow\left\langle\widehat{Q}_{B} \Psi_{m}\right| b_{0}^{+}=0,
$$

where in the last step we have used (2.7) and the fact that $b_{0}^{-} \widehat{Q}_{B}\left|\Psi_{m}\right\rangle=0$. (2.23) is just a reflection of the fact that the poles of $\Delta$ are associated with zero eigenvalues of the kinetic operator in the Siegel gauge.

We now classify the candidates for $\left|\Phi_{m}\right\rangle$ satisfying these conditions near a particular pole.

1. Unphysical states: these are linearly independent states $\left|U_{r}\right\rangle$ satisfying

$$
b_{0}^{+}\left|U_{r}\right\rangle=0, \quad b_{0}^{+} \widehat{Q}_{B}\left|U_{r}\right\rangle=0, \quad \widehat{Q}_{B} \sum_{r} a_{r}\left|U_{r}\right\rangle \neq 0,
$$

for any choice of $\left\{a_{r}\right\}$ other than $a_{r}=0$ for every $r$.

2. Physical states: these are states satisfying

$$
b_{0}^{+}\left|P_{a}\right\rangle=0, \quad \widehat{Q}_{B}\left|P_{a}\right\rangle=0, \quad \sum_{a} c_{a}\left|P_{a}\right\rangle \neq \sum_{r} d_{r} \widehat{Q}_{B}\left|U_{r}\right\rangle
$$

for any choice of $\left\{c_{a}\right\},\left\{d_{r}\right\}$ other than $c_{a}=0$ for every $a$ and $d_{r}=0$ for every $r$.

3. Pure gauge states: these are states of the form $\widehat{Q}_{B}\left|U_{r}\right\rangle$. These are automatically annihilated by $\widehat{Q}_{B}$ due to $(2.6)$ and by $b_{0}^{+}$due to $(2.24)$.

Note that for any given momentum if there is an unphysical state $\left|U_{r}\right\rangle$, there is also a pure gauge state $\widehat{Q}_{B}\left|U_{r}\right\rangle$. Generically we expect no other degeneracy but we shall proceed without making this assumption. A similar classification can be done for the candidates for $\left\langle\Psi_{m}\right|$.

Let us now suppose that at some given momentum at which $\Delta$ has pole, there are a certain number of linearly independent physical states $\left\{\left|P_{a}\right\rangle\right\}$, unphysical states $\left\{\left|U_{r}\right\rangle\right\}$ and pure gauge states $\left\{\widehat{Q}_{B}\left|U_{r}\right\rangle\right\}$. The normalization of these states is chosen arbitrarily. Then the general form of $\Delta_{0}$ is given by

$$
\Delta_{0}=\sum_{a}\left|P_{a}\right\rangle\left\langle B_{a}\left|+\sum_{r}\right| U_{r}\right\rangle\left\langle C_{r}\left|+\sum_{r} \widehat{Q}_{B}\right| U_{r}\right\rangle\left\langle D_{r}\right|,
$$


for some states $\left|B_{a}\right\rangle,\left|C_{r}\right\rangle,\left|D_{r}\right\rangle \in \widehat{\mathcal{H}}_{T}$. Eq. (2.23) now gives

$$
\langle\Psi| b_{0}^{+}=0, \quad\left\langle\widehat{Q}_{B} \Psi\right| b_{0}^{+}=0, \quad \text { for }\langle\Psi|=\left\langle B_{a}\right|,\left\langle C_{r}\right| \text { or }\left\langle D_{r}\right| .
$$

Substituting (2.26) into (2.19) and using (2.24), (2.25) we also get

$$
\sum_{r} \widehat{Q}_{B}\left|U_{r}\right\rangle\left\langle C_{r}\left|c_{0}^{-}+\sum_{a}\right| P_{a}\right\rangle\left\langle B_{a}\left|c_{0}^{-} \widetilde{Q}_{B}+\sum_{r}\right| U_{r}\right\rangle\left\langle C_{r}\left|c_{0}^{-} \widetilde{Q}_{B}+\sum_{r} \widehat{Q}_{B}\right| U_{r}\right\rangle\left\langle D_{r}\right| c_{0}^{-} \widetilde{Q}_{B}=0 .
$$

Using this, and the fact that $\left\{\left|P_{a}\right\rangle\right\},\left\{\left|U_{r}\right\rangle\right\}$ and $\left\{\widehat{Q}_{B}\left|U_{r}\right\rangle\right\}$ are linearly independent, we get

$$
\begin{aligned}
\left\langle B_{a}\right| c_{0}^{-} \widetilde{Q}_{B}=0 & \Rightarrow\left\langle\widehat{Q}_{B} B_{a}\right|=0, \\
\left\langle C_{r}\right| c_{0}^{-}=-\left\langle D_{r}\right| c_{0}^{-} \widetilde{Q}_{B} & \Rightarrow \quad\left\langle C_{r}\right|=-(-1)^{D_{r}}\left\langle\widehat{Q}_{B} D_{r}\right|, \\
\left\langle C_{r}\right| c_{0}^{-} \widetilde{Q}_{B}=0 & \Rightarrow\left\langle\widehat{Q}_{B} C_{r}\right|=0 .
\end{aligned}
$$

$(-1)^{D_{r}}$ takes value 1 if $D_{r}$ is grassmann even and -1 if $D_{r}$ is grassmann odd. The last equation in (2.29) in fact follows from the second equation and nilpotence of $\widetilde{Q}_{B}$. Using the second equation in $(2.29)$ we can rewrite $(2.26)$ as

$$
\Delta_{0}=\sum_{a}\left|P_{a}\right\rangle\left\langle B_{a}\left|-\sum_{r}(-1)^{D_{r}}\right| U_{r}\right\rangle\left\langle\widehat{Q}_{B} D_{r}\left|+\sum_{r} \widehat{Q}_{B}\right| U_{r}\right\rangle\left\langle D_{r}\right| .
$$

Since according to (2.8) $\Delta$ carries total ghost number -2 and since the BPZ inner product pairs states carrying total ghost number 6 , we have

$$
n_{P_{a}}+n_{B_{a}}=4, \quad n_{U_{r}}+n_{D_{r}}=3
$$

where for any state $|A\rangle, n_{A}$ denotes its ghost number.

The generalization of this analysis to type II string theories is straightforward [15]. The string field will now have four sectors satisfying NSNS, NSR, RNS and RR boundary conditions. $\mathcal{G}$ will be given by the identity operator in the NSNS sector, zero mode of the right-handed PCO in the NSR sector, zero mode of the left-handed PCO in the RNS sector and the product of the zero modes of the left-handed and right-handed PCO's in the RR sector. $\widehat{\mathcal{H}}_{T}$ and $\widetilde{\mathcal{H}}_{T}$ will be defined as

$$
\begin{aligned}
& \widehat{\mathcal{H}}_{T}=\mathcal{H}_{-1,-1} \oplus \mathcal{H}_{-1,-1 / 2} \oplus \mathcal{H}_{-1 / 2 .-1} \oplus \mathcal{H}_{-1 / 2,-1 / 2}, \\
& \widetilde{\mathcal{H}}_{T}=\mathcal{H}_{-1,-1} \oplus \mathcal{H}_{-1,-3 / 2} \oplus \mathcal{H}_{-3 / 2,-1} \oplus \mathcal{H}_{-3 / 2,-3 / 2}
\end{aligned}
$$

where $\mathcal{H}_{m, n}$ denotes the subspace of $\mathcal{H}_{T}$ carrying left-handed picture number $m$ and righthanded picture number $n$. The rest of the analysis remains unchanged.

\section{Unitarity}

In this section we shall prove unitarity of the amplitudes of superstring field theory. This analysis will be valid for both heterotic and type II string theories. 


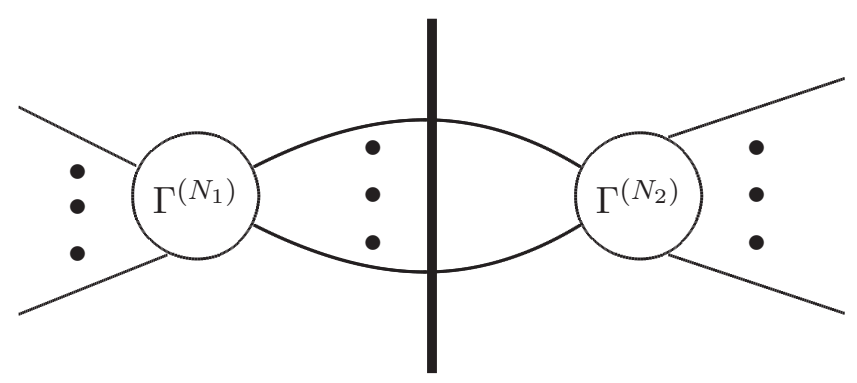

Figure 4. A cut diagram in superstring field theory.

Let $\Gamma^{(N)}\left(\left|A_{1}\right\rangle, \cdots\left|A_{N}\right\rangle\right)$ denote the truncated Green's function in which the external leg propagators are removed. $\Gamma^{(N)}$ satisfies the following Ward identity [15]:

$$
\sum_{i=1}^{N}(-1)^{\gamma_{1}+\cdots \gamma_{i-1}} \Gamma^{(N)}\left(\left|A_{1}\right\rangle, \cdots\left|A_{i-1}\right\rangle, \widehat{Q}_{B}\left|A_{i}\right\rangle,\left|A_{i+1}\right\rangle, \cdots\left|A_{N}\right\rangle\right)=0,
$$

where $\gamma_{i}$ is the grassmannality of $A_{i}$.

Now in a cut diagram like the one shown in figure 4 , each cut propagator is replaced by the right hand side of (2.30) together with a $2 \pi \delta\left(k^{2}+m^{2}\right) \theta\left(k^{0}\right)$ factor. Let us suppose that we have a cut diagram with $N$ cut propagators. Using the superscript $(i)$ to label the states associated with the $i$-th cut propagator and the operators acting on these states, we have a net factor of

$$
\prod_{i=1}^{N}\left(\Delta_{0}\right)^{(i)}=\prod_{i=1}^{N}\left[\sum_{a}\left|P_{a}^{(i)}\right\rangle\left\langle B_{a}^{(i)}\left|-\sum_{r}(-1)^{D_{r}^{(i)}}\right| U_{r}^{(i)}\right\rangle\left\langle\widehat{Q}_{B}^{(i)} D_{r}^{(i)}\left|+\sum_{r} \widehat{Q}_{B}^{(i)}\right| U_{r}^{(i)}\right\rangle\left\langle D_{r}^{(i)}\right|\right],
$$

associated with all the cut propagators. ${ }^{5}$ In this the ket states are inserted into the amplitude $\Gamma^{\left(N_{1}\right)}$ on the left side of the cut and the bra states are inserted into the amplitude $\Gamma^{\left(N_{2}\right)}$ on the right side of the cut. Besides these $\Gamma^{\left(N_{1}\right)}$ and $\Gamma^{\left(N_{2}\right)}$ have insertions of external incoming and outgoing states respectively, which are all annihilated by $\widehat{Q}_{B}$.

We now expand (3.2) as a sum of $3^{N}$ terms. There is one term given by

$$
\prod_{i=1}^{N}\left\{\sum_{a}\left|P_{a}^{(i)}\right\rangle\left\langle B_{a}^{(i)}\right|\right\} \text {. }
$$

Each of the other terms has a certain number (say $K<N$ ) of factors of $\sum_{a}\left|P_{a}^{(i)}\right\rangle\left\langle B_{a}^{(i)}\right|$. We group together all terms with the same factors of $\sum_{a}\left|P_{a}^{(i)}\right\rangle\left\langle B_{a}^{(i)}\right|$, and in any given group we denote by $S$ the set of labels $i$ carried by the rest of the factors. $S$ contains $N-K$ elements. We separate out from $S$ one particular label which we call $\alpha$. For definiteness we can take $\alpha$ to be the lowest element of $S$. For any $A \subseteq S-\{\alpha\}$, where $S_{1}-S_{2}$ for $S_{2} \subseteq S_{1}$ denotes the set $S_{1}$ with the elements in $S_{2}$ removed, let us denote by $F_{S}(\alpha ; A)$ the amplitude associated with the cut diagram where the label $\alpha$ is carried by the factor $-\sum_{r}(-1)^{D_{r}^{(\alpha)}}\left|U_{r}^{(\alpha)}\right\rangle\left\langle\widehat{Q}_{B}^{(\alpha)} D_{r}^{(\alpha)}\right|$, the labels $i$ in $A$ are carried by $-\sum_{r}(-1)^{D_{r}^{(i)}}\left|U_{r}^{(i)}\right\rangle\left\langle\widehat{Q}_{B}^{(i)} D_{r}^{(i)}\right|$ and the labels $i$ in $S-\{\alpha\}-A$ are carried by

\footnotetext{
${ }^{5}$ The range of $a$ and $r$ in (3.2) are in general different for different $i$.
} 
$\sum_{r} \widehat{Q}_{B}^{(i)}\left|U_{r}^{(i)}\right\rangle\left\langle D_{r}^{(i)}\right|$. Similarly we denote by $G_{S}(\alpha ; A)$ the amplitude where the label $\alpha$ is carried by $\sum_{r} \widehat{Q}_{B}^{(\alpha)}\left|U_{r}^{(\alpha)}\right\rangle\left\langle D_{r}^{(\alpha)}\right|$, the labels $i$ in $A$ are carried by $-\sum_{r}(-1)^{D_{r}^{(i)}}\left|U_{r}^{(i)}\right\rangle\left\langle\widehat{Q}_{B}^{(i)} D_{r}^{(i)}\right|$ and the labels $i$ in $S-\{\alpha\}-A$ are carried by $\sum_{r} \widehat{Q}_{B}^{(i)}\left|U_{r}^{(i)}\right\rangle\left\langle D_{r}^{(i)}\right|$. Then the sum of all terms in a given group, i.e. with a fixed set of labels $i$ carrying $\sum_{a}\left|P_{a}^{(i)}\right\rangle\left\langle B_{a}^{(i)}\right|$ factors, is given by

$$
\sum_{A \subseteq S-\{\alpha\}}\left[F_{S}(\alpha ; A)+G_{S}(\alpha ; A)\right] .
$$

Let us consider the amplitude $F_{S}(\alpha ; A)$. In this case the insertion associated with the line $\alpha$ to the amplitude $\Gamma^{\left(N_{2}\right)}$ on the right of the cut is $-(-1)^{D_{r}^{(\alpha)}}\left\langle\widehat{Q}_{B}^{(\alpha)} D_{r}^{(\alpha)}\right|$. The other insertions involve the states $\left\langle B_{a}^{(i)}\right|$ for $i \notin S$, the states $-(-1)^{D_{r}^{(i)}}\left\langle\widehat{Q}_{B}^{(i)} D_{r}^{(i)}\right|$ with $i \in A$, the states $\left\langle D_{r}^{(i)}\right|$ for $i \in S-\{\alpha\}-A$ and the external physical states which are all annihilated by $\widehat{Q}_{B}$. Now we can use (3.1) to express this amplitude as a sum of terms in which $\widehat{Q}_{B}^{(\alpha)} D_{r}^{(\alpha)}$ is replaced by $D_{r}^{(\alpha)}$, but $\widehat{Q}_{B}$ acts in turn on the other states. Since the external states as well as $B_{a}^{(i)}$ and $\widehat{Q}_{B}^{(i)} D^{(i)}$ are all annihilated by $\widehat{Q}_{B}$, the only non-vanishing contribution comes from the terms where $\widehat{Q}_{B}$ acts on one of the states $\left\langle D_{r}^{(j)}\right|$ for $j \in S-\{\alpha\}-A$. This gives,

$$
F_{S}(\alpha ; A)=\sum_{j \in S-\{\alpha\}-A} s(\alpha ; j ; A) H_{S}(\alpha ; j ; A)
$$

where $s(\alpha ; j ; A)$ takes value \pm 1 and $H_{S}(\alpha ; j ; A)$ denotes an amplitude where the label $\alpha$ is carried by $\sum_{r}\left|U_{r}^{(\alpha)}\right\rangle\left\langle D_{r}^{(\alpha)}\right|$, the label $j$ is carried by $-\sum_{r}(-1)^{D_{r}^{(j)}} \widehat{Q}_{B}^{(j)}\left|U_{r}^{(j)}\right\rangle\left\langle\widehat{Q}_{B}^{(j)} D_{r}^{(j)}\right|$, the labels $i$ in $A$ are carried by $-\sum_{r}(-1)^{D_{r}^{(i)}}\left|U_{r}^{(i)}\right\rangle\left\langle\widehat{Q}_{B}^{(i)} D_{r}^{(i)}\right|$ and the labels $i$ in $S-\{\alpha\}-A-\{j\}$ are carried by $\sum_{r} \widehat{Q}_{B}^{(i)}\left|U_{r}^{(i)}\right\rangle\left\langle D_{r}^{(i)}\right|$. Carrying out a similar manipulation of the amplitude $\Gamma^{\left(N_{1}\right)}$ on the left of the cut, we get

$$
G_{S}(\alpha ; A)=\sum_{j \in A} s^{\prime}(\alpha ; j ; A-\{j\}) H_{S}(\alpha ; j ; A-\{j\}) .
$$

where $s^{\prime}(\alpha ; j ; A-\{j\})$ takes value \pm 1 . This gives

$$
\begin{aligned}
\sum_{A \subseteq S-\{\alpha\}} F_{S}(\alpha ; A) & =\sum_{A \subseteq S-\{\alpha\}} \sum_{j \in S-A-\{\alpha\}} s(\alpha ; j ; A) H_{S}(\alpha ; j ; A) \\
& =\sum_{j \in S-\{\alpha\}} \sum_{A \subseteq S-\{\alpha, j\}} s(\alpha ; j ; A) H_{S}(\alpha ; j ; A),
\end{aligned}
$$

and

$$
\begin{aligned}
\sum_{A \subseteq S-\{\alpha\}} G_{S}(\alpha ; A) & =\sum_{A \subseteq S-\{\alpha\}} \sum_{j \in A} s^{\prime}(\alpha ; j ; A-\{j\}) H_{S}(\alpha ; j ; A-\{j\}) \\
& =\sum_{j \in S-\{\alpha\}} \sum_{A \subseteq S-\{\alpha, j\}} s^{\prime}(\alpha ; j ; A) H_{S}(\alpha ; j ; A),
\end{aligned}
$$

where in the last step we have relabelled $A-\{j\}$ as $A$. The right hand sides of (3.7) and (3.8) are the same up to signs. We shall now show that the signs are such that these terms cancel pairwise in (3.4). 
The manipulations in (3.7) involve the rearrangement

$$
\begin{gathered}
-(-1)^{D_{r}^{(\alpha)}}\left|U_{r}^{(\alpha)}\right\rangle\left\langle\widehat{Q}_{B}^{(\alpha)} D_{r}^{(\alpha)}\left|\left\{\prod_{i ; \alpha<i<j}\left(\Delta_{0}\right)^{(i)}\right\} \widehat{Q}_{B}^{(j)}\right| U_{r}^{(j)}\right\rangle\left\langle D_{r}^{(j)}\right| \\
\Rightarrow(-1)^{D_{r}^{(j)}}\left|U_{r}^{(\alpha)}\right\rangle\left\langle D_{r}^{(\alpha)}\left|\left\{\prod_{i ; \alpha<i<j}\left(\Delta_{0}\right)^{(i)}\right\} \widehat{Q}_{B}^{(j)}\right| U_{r}^{(j)}\right\rangle\left\langle\widehat{Q}_{B}^{(j)} D_{r}^{(j)}\right| .
\end{gathered}
$$

The sign on the right hand side is fixed as follows. First there is a minus sign from having to take all but one term from the left to the right hand side of (3.1). Second there is a factor of $(-1)^{D_{r}^{(\alpha)}}$ from having to take $\widehat{Q}_{B}$ through $D_{r}^{(\alpha)}$. These two together cancel the $-(-1)^{D_{r}^{(\alpha)}}$ factor on the left. Moving $\widehat{Q}_{B}$ through $\prod_{i}\left(\Delta_{0}\right)^{(i)}$ does not generate a sign since the latter operator is grassmann even. ${ }^{6}$ Finally moving $\widehat{Q}_{B}$ through $\widehat{Q}_{B}^{(j)}\left|U_{r}^{(j)}\right\rangle$ generates a factor of $-(-1)^{U_{r}^{(j)}}=(-1)^{D_{r}^{(j)}}$ using (2.31). This is the factor we see on the right hand side of (3.9).

On the other hand manipulations in (3.8) involve the rearrangement

$$
\begin{aligned}
& -(-1)^{D_{r}^{(j)}} \widehat{Q}_{B}^{(\alpha)}\left|U_{r}^{(\alpha)}\right\rangle\left\langle D_{r}^{(\alpha)}\left|\left\{\prod_{i ; \alpha<i<j}\left(\Delta_{0}\right)^{(i)}\right\}\right| U_{r}^{(j)}\right\rangle\left\langle\widehat{Q}_{B}^{(j)} D_{r}^{(j)}\right| \\
& \Rightarrow-(-1)^{D_{r}^{(j)}}\left|U_{r}^{(\alpha)}\right\rangle\left\langle D_{r}^{(\alpha)}\left|\left\{\prod_{i ; \alpha<i<j}\left(\Delta_{0}\right)^{(i)}\right\} \widehat{Q}_{B}^{(j)}\right| U_{r}^{(j)}\right\rangle\left\langle\widehat{Q}_{B}^{(j)} D_{r}^{(j)}\right| .
\end{aligned}
$$

In this manipulation two minus signs cancel. First of all we get a minus sign from having to take all but one term in (3.1) from the left to the right side. Since $\left|U_{r}^{(\alpha)}\right\rangle\left\langle D_{r}^{(\alpha)}\right|$ is a grassmann odd operator due to (2.31), passing $\widehat{Q}_{B}$ through this generates a second minus sign. Therefore the right hand side of (3.10) has the same sign as the left hand side.

We now see that the the right hand sides of (3.9) and (3.10) cancel. This cancelation works for every term in (3.7) and (3.8), making (3.4) vanish. This shows that the only term that contributes is the one where (3.2) is replaced by (3.3).

This still does not prove that only physical states contribute since the only information about $\left\langle B_{a}\right|$ that we have is from (2.27) and the first equation in (2.29), and this allows $\left\langle B_{a}\right|$ to be either a physical state or a pure gauge state of the form $\left\langle\widehat{Q}_{B} E_{a}\right|$ for some $\left\langle E_{a}\right|$. However since all other states entering in the argument of $\Gamma^{\left(N_{2}\right)}$ are annihilated by $\widehat{Q}_{B}$, the amplitude with one or more $\left\langle B_{a}\right|$ having the form $\left\langle\widehat{Q}_{B} E_{a}\right|$ will vanish due to (3.1). This shows that $\left\langle B_{a}\right|$ must be a physical state. It now follows from (3.3) that only physical states contribute to the cut propagators. This is the desired result that establishes unitarity of the amplitude.

\footnotetext{
${ }^{6}$ Note that we are not actually commuting $\widehat{Q}_{B}$ through the operators $\Delta^{(i)}$, — this would generate additional terms due to (2.10). The transfer of $\widehat{Q}_{B}$ from one state to another takes place through the amplitude $\Gamma^{\left(N_{2}\right)}$. However the extra sign picked up due to the grassmannality of the operators can be determined just from the relative position of the operators in an expression, and that is the way we are determining the sign.
} 


\section{Discussions}

In this paper we have completed the proof of unitarity of covariant superstring field theory. Therefore this theory represents a manifestly Lorentz invariant, ultraviolet finite and unitary theory. Furthermore infrared divergences associated with tadpoles and mass renormalization can be dealt with using standard quantum field theory techniques.

We must note however that when the number of non-compact space-time dimensions $D$ is 4 or less, the S-matrix suffers from the usual infrared divergences and we have to carry out the usual procedure of summing over final states and averaging over initial states to get a finite result for physical cross section [21-24]. This has not yet been worked out in superstring field theory. We hope to return to this problem in the future.

\section{Acknowledgments}

I wish to thank Roji Pius for useful discussions. This work was supported in part by the DAE project 12-R\&D-HRI-5.02-0303 and J. C. Bose fellowship of the Department of Science and Technology, India.

Open Access. This article is distributed under the terms of the Creative Commons Attribution License (CC-BY 4.0), which permits any use, distribution and reproduction in any medium, provided the original author(s) and source are credited.

\section{References}

[1] R. Pius and A. Sen, Cutkosky rules for superstring field theory, JHEP 10 (2016) 024 [arXiv: 1604.01783] [INSPIRE].

[2] A. Sen, Reality of superstring field theory action, JHEP 11 (2016) 014 [arXiv:1606.03455] [INSPIRE].

[3] S.B. Giddings, Conformal techniques in string theory and string field theory, Phys. Rept. 170 (1988) 167 [INSPIRE].

[4] K. Aoki, E. D'Hoker and D.H. Phong, Unitarity of closed superstring perturbation theory, Nucl. Phys. B 342 (1990) 149 [inSPIRE].

[5] S. Mandelstam, Interacting string picture of dual resonance models, Nucl. Phys. B 64 (1973) 205 [inSPIRE].

[6] S. Mandelstam, Interacting string picture of the Neveu-Schwarz-Ramond model, Nucl. Phys. B 69 (1974) 77 [InSPIRE].

[7] J. Greensite and F.R. Klinkhamer, New interactions for superstrings, Nucl. Phys. B 281 (1987) 269 [inSPIRE].

[8] J. Greensite and F.R. Klinkhamer, Contact interactions in closed superstring field theory, Nucl. Phys. B 291 (1987) 557 [inSPIRE].

[9] J. Greensite and F.R. Klinkhamer, Superstring amplitudes and contact interactions, Nucl. Phys. B 304 (1988) 108 [inSPIRE]. 
[10] M.B. Green and N. Seiberg, Contact interactions in superstring theory, Nucl. Phys. B 299 (1988) 559 [inSPIRE].

[11] E. Witten, Superstring perturbation theory revisited, arXiv:1209.5461 [INSPIRE].

[12] N. Ishibashi, Light-cone gauge superstring field theory in linear dilaton background, arXiv: 1605.04666 [INSPIRE].

[13] A. Berera, Unitary string amplitudes, Nucl. Phys. B 411 (1994) 157 [InSPIRE].

[14] E. Witten, The Feynman i€ in string theory, JHEP 04 (2015) 055 [arXiv:1307.5124] [INSPIRE].

[15] A. Sen, Supersymmetry restoration in superstring perturbation theory, JHEP 12 (2015) 075 [arXiv: 1508.02481] [INSPIRE].

[16] A. Sen, BV master action for heterotic and type II string field theories, JHEP 02 (2016) 087 [arXiv: 1508.05387] [INSPIRE].

[17] B. Zwiebach, Closed string field theory: quantum action and the B-V master equation, Nucl. Phys. B 390 (1993) 33 [hep-th/9206084] [INSPIRE].

[18] R. Pius, A. Rudra and A. Sen, Mass renormalization in string theory: general states, JHEP 07 (2014) 062 [arXiv: 1401.7014] [INSPIRE].

[19] M.J.G. Veltman, Unitarity and causality in a renormalizable field theory with unstable particles, Physica 29 (1963) 186 [INSPIRE].

[20] G. 't Hooft and M.J.G. Veltman, Diagrammar, NATO Sci. Ser. B 4 (1974) 177 [InSPIRE].

[21] T. Kinoshita, Mass singularities of Feynman amplitudes, J. Math. Phys. 3 (1962) 650 [INSPIRE].

[22] T.D. Lee and M. Nauenberg, Degenerate systems and mass singularities, Phys. Rev. 133 (1964) B1549 [inSPIRE].

[23] F. Bloch and A. Nordsieck, Note on the radiation field of the electron, Phys. Rev. 52 (1937) 54 [INSPIRE].

[24] G.F. Sterman, An introduction to quantum field theory, Cambridge University Press, Cambridge U.K. (1993) [inSPIRE]. 